\title{
Online failure probability estimation under state estimation error and its application to angle of attack control of a reentry vehicle
}

\author{
Nicolas Merlinge, Thibault Cantou, Karim Dahia \\ ONERA - The French Aerospace Lab
}

\begin{abstract}
Knowing the probability of failure in real time i.e. the probability that a system violates some constraints is decisive for aerospace applications in the presence of state uncertainty. However, it often involves stochastic prediction of the constraints on the upcoming system's trajectory, which is computationally demanding. In addition, the trajectory prediction depends on the accuracy of the current state estimate, e.g. the navigation system, whose errors are usually not accounted for. This paper studies the impact of the state estimate error on the failure probability calculation, obtained by Monte Carlo trajectory sampling. The failure probability error is first shown to be made of two terms depending on the current state estimation error and the number of Monte Carlo samples. Then, an iterative Least Square estimator is introduced to refine the failure probability estimation without significantly increasing the computational load. It is theoretically shown to converge and lowers the failure probability estimation error of $33 \%$ in simulation. The approach is illustrated on a constrained stochastic control application: the atmospheric reentry of a vehicle inspired from SpaceX's Starship (SXS). The proposed method allows the failure probability estimate to be more accurate despite the unavoidable disturbances yielded by the state estimation algorithm and the Monte Carlo discrete sampling, in particular when the available computational load is limited onboard.
\end{abstract}

\section{INTRODUCTION}

Estimating the probability that a system breaks up or fails is crucial for decision and control applications. This problem was widely addressed for offline risk of failure analysis of critical systems [1], [2], [3], [4]. Knowing the failure probability is also essential for real time applications in the presence of uncertainties. Online failure probability estimation was mostly addressed for stochastic predictive control applications [5], [6], [7], [8], [9], [10]. These approaches require the knowledge of the current state density. In practice, the system's state density is provided by a state estimator which may be temporarily biased or noisy. This paper addresses online failure probability estimation under input uncertainty, state estimation error, and computational load constraint.

Consider an evolving dynamical system defined by its state. The state is a stochastic process that can only be measured by some stochastic observation (e.g. sensor measurements). A common way to estimate the system's state is using Bayesian filtering (e.g. Extended Kalman Filter [11]). Bayesian filters aim to retrieve the current state density given the past measurements. To predict the future stochastic behavior of the system, the state density can be propagated upon a given time-horizon, which yields a trajectory density. The future trajectory density can be formalized as a Markov Chain [12]. The prediction of the probability that a system fails in a near future can be formalized as a probability measure of the future trajectory density on a failure domain - or constraint domain.

Analytic formulations of the failure probability were proposed for given state densities and linear dynamics [7], [8]. However, for non-linear dynamics and/or non-linear state constraints, the trajectory density cannot be formalized analytically. Numerical approximations were introduced, e.g. the Monte Carlo based approaches [13]. These approaches consist in sampling a finite number of future trajectories and counting how many bring the system to a failure - i.e violate some state constraints. Several methods were derived from the Monte Carlo density sampling approach to approximate the failure probability [14]. The accuracy of these methods grows with the number of trajectory samples. An error may rise in the failure probability estimation when the number of samples is limited for computational reasons.

In this paper, Monte Carlo failure probability estimation is addressed in the context of erratic state estimate. Section II describes the probabilistic framework of the problem. An application example to constrained control is introduced. Section III addresses two questions:

- How does the Monte Carlo approximation of the failure probability behave in the presence of state estimation error? (Section III-A)

- Is it possible to refine the failure probability estimation? An iterative Least Square estimator is introduced and proved to converge to the actual failure probability (Section III-B).

Section IV provides two application examples for a state constrained control problem of a Starship-like spacecraft [15]. Section V illustrates the performances of the proposed approach and Section VI concludes the work.

\section{PROBLEM STATEMENT}

Consider the following non-linear discrete-time dynamical model with state $\mathbf{x}_{k} \in \mathbb{R}^{d}$ and control input $\mathbf{u}_{k} \in \mathbb{R}^{d_{c}}$ $(k \in \mathbb{N})$ :

$$
\mathbf{x}_{k}=f\left(\mathbf{x}_{k-1}, \mathbf{u}_{k}\right)+\mathbf{w}_{k}
$$

where $\mathbf{w}_{k}$ is a stochastic process noise. The random process $\mathbf{x}_{k}$ cannot be directly measured, but must be retrieved by a 
related measurement $\mathbf{y}_{k} \in \mathbb{R}^{d_{y}}$ :

$$
\mathbf{y}_{k}=h\left(\mathbf{x}_{k-1}\right)+\mathbf{v}_{k}
$$

where $\mathbf{v}_{k}$ is a stochastic measurement noise. Define some output vector $\mathbf{z}_{k} \in \mathbb{R}^{d_{z}}$ :

$$
\mathbf{z}_{k}=g\left(\mathbf{x}_{k-1}\right)
$$

where $g$ is a potentially non-linear $\mathcal{C}^{0}$ application. Define a trajectory prediction application $F$ that takes the current state $\mathbf{x}_{k}$ and outputs a future state trajectory from time-step $k$ to time-step $K>k$. Time $K$ can be a fixed term horizon or a receding horizon, i.e $K=k+\Delta k\left(\Delta k \in \mathbb{R}^{+*}\right)$.

$$
\begin{aligned}
& F_{K}: \mathbb{R}^{d} \times \mathbb{R}^{d_{c}(K-k+1)} \times \mathbb{R}^{d(K-k+1)} \rightarrow \mathbb{R}^{d(K-k+1)} \\
&\left(\mathbf{x}_{k}, \mathbf{u}_{k: K}, \mathbf{w}_{k: K}\right) \mapsto \mathbf{x}_{k: K} \triangleq\left[\mathbf{x}_{k}^{T}, \ldots, \mathbf{x}_{K}^{T}\right]^{T}
\end{aligned}
$$

where the predicted states $\mathbf{x}_{i} \in \mathbb{R}^{d}$ and process noises $\mathbf{w}_{k: K} \triangleq\left[\mathbf{w}_{k}^{T}, \ldots, \mathbf{w}_{K}^{T}\right]^{T}$ are obtained using (1), for a given future control sequence $\mathbf{u}_{k: K} \triangleq\left[\mathbf{u}_{k}^{T}, \ldots, \mathbf{u}_{K}^{T}\right]^{T}$. Subsequently, define the output trajectory $\mathbf{z}_{k: K} \triangleq\left[\mathbf{z}_{k}^{T}, \ldots, \mathbf{z}_{K}^{T}\right]^{T}$. This paper tackles the issue of determining the probability $p_{f_{k}}$ that $\mathbf{z}_{k: K}$ exceeds some threshold $\overline{\mathbf{Z}}=\left[\overline{\mathbf{z}}_{k}^{T}, \ldots, \overline{\mathbf{z}}_{K}^{T}\right]^{T} \in$ $\mathbb{R}^{d_{z}(K-k+1)}$ :

$$
p_{f_{k}} \triangleq \mathbb{P}\left(\mathbf{z}_{k: K}>\overline{\mathbf{Z}}\right)
$$

The probability $p_{f}$ is referred to as failure probability. In the following of this section, we introduce the probabilistic framework describing the dynamics, measurements, and outputs, as well as a sample-based approximation of $p_{f}$. An example of application to control under uncertainty is also introduced.

\section{A. Probabilistic scheme}

In probabilistic schemes, the process and measurement noises are associated with some probability density function: the transition density $p\left(\mathbf{x}_{k} \mid \mathbf{x}_{k-1}\right)$ and the likelihood $p\left(\mathbf{y}_{k} \mid \mathbf{x}_{k}\right)$. In this subsection, we first present the state estimation framework to approach the current state $\mathbf{x}_{k}$. We then introduce a probabilistic description of the predicted trajectory of outputs.

1) State estimation: Bayesian state estimation is performed in two iterative steps. The time-update step derives the predicted state density from the prior state density $p\left(\mathbf{x}_{k-1} \mid \mathbf{Y}_{k-1}\right)$ (where $\left.\mathbf{Y}_{k}=\left\{\mathbf{y}_{1}, \ldots, \mathbf{y}_{k}\right\}\right)$ and the transition density $p\left(\mathbf{x}_{k} \mid \mathbf{x}_{k-1}\right)$ :

$$
p\left(\mathbf{x}_{k} \mid \mathbf{Y}_{k-1}\right)=\int p\left(\mathbf{x}_{k} \mid \mathbf{x}_{k-1}\right) p\left(\mathbf{x}_{k-1} \mid \mathbf{Y}_{k-1}\right) \mathrm{d} \mathbf{x}_{k-1}
$$

The measurement-update step retrieves the posterior state density from the predicted state density and the measurements density $p\left(\mathbf{y}_{k} \mid \mathbf{x}_{k}\right)$ :

$$
p\left(\mathbf{x}_{k} \mid \mathbf{Y}_{k}\right)=\frac{p\left(\mathbf{y}_{k} \mid \mathbf{x}_{k}\right) p\left(\mathbf{x}_{k} \mid \mathbf{Y}_{k-1}\right)}{\int p\left(\mathbf{y}_{k} \mid \mathbf{x}_{k}\right) p\left(\mathbf{x}_{k} \mid \mathbf{Y}_{k-1}\right) \mathrm{d} \mathbf{x}_{k}}
$$

In the linear-Gaussian case, the Kalman Filter is shown to be optimal in the sense of (6) and (7). For nonlinear dynamics (1) and/or measurements (2), Bayesian filters may produce an erratic posterior state density estimate $\widehat{p}\left(\mathbf{x}_{k} \mid \mathbf{Y}_{k}\right)$, which will impact the failure probability evaluation.
2) Output trajectory density: The predicted state trajectory (4) is a Markov Chain [12], then the trajectory density is:

$$
p\left(\mathbf{x}_{k: K} \mid \mathbf{Y}_{k}\right)=p\left(\mathbf{x}_{k} \mid \mathbf{Y}_{k}\right) \prod_{i=k+1}^{K} p\left(\mathbf{x}_{i} \mid \mathbf{x}_{i-1}\right)
$$

Since output $\mathbf{z}_{k}$ depends on $\mathbf{x}_{k}$ for all $k$, the output trajectory $\mathbf{z}_{k: K}$ is also a Markov process whose density can be derived from (8): $p\left(\mathbf{z}_{k: K} \mid \mathbf{Y}_{k}\right)=p\left(\mathbf{z}_{k} \mid \mathbf{Y}_{k}\right) \prod_{i=k+1}^{K} p\left(\mathbf{z}_{i} \mid \mathbf{z}_{i-1}\right)$.

3) Monte Carlo failure probability estimation: Define $\mu_{\pi}(\mathcal{A})$ the measure of density $\pi$ on domain $\mathcal{A}$ :

$$
\mu_{\pi}(\mathcal{A}) \triangleq \int \mathbf{1}_{\mathcal{A}} \pi(\mathbf{x}) d \mathbf{x}
$$

where $\mathbf{1}_{\mathcal{A}}(\mathbf{x})=1$ if $\mathbf{x} \in \mathcal{A}$ and $\mathbf{1}_{\mathcal{A}}(\mathbf{x})=0$ elsewhere. The failure probability (5) can be expressed by:

$$
p_{f_{k}}=\mu_{p\left(\mathbf{z}_{k: K} \mid \mathbf{Y}_{k}\right)}\left(\left\{\mathbf{z}_{k: K}>\overline{\mathbf{Z}}\right\}\right)
$$

Since $\mathbf{z}_{k: K}$ is obtained by a nonlinear recursive prediction using the dynamical model (1) and a non-linear output application $g(3)$, the analytic solution is intractable. A way to approximate $p_{f_{k}}$ is simulating $N$ sampled output trajectories $\left\{\mathbf{z}_{k: K}^{i}\right\}_{i=\{1, \ldots, N\}}$ drawn from the current state estimate density $\widehat{p}\left(\mathbf{x}_{k} \mid \mathbf{Y}_{k}\right)$ :

$$
p_{f_{k}}^{N}=\frac{1}{N} \sum_{i=1}^{N} \mathbf{1}_{\mathbf{z}_{k: K}>\overline{\mathbf{Z}}}\left(\mathbf{z}_{k: K}^{i}\right)
$$

This approach is called Monte Carlo probability measure approximation. It can be shown that $\lim _{k \rightarrow \infty}\left(p_{f_{k}}^{N}\right)=p_{f_{k}}$. However, the algorithmic cost of $\mathbf{z}_{k: K}$, necessary to compute (11), is about $\mathcal{O}\left(N(K-k)\left(c_{f}+c_{g}\right)\right)$ in terms of elementary operations (additions, subtraction, multiplications and divisions). Values $c_{f}>0$ and $c_{g}>0$ denote the cost of the dynamical transition $f$ and the output computation $g$ respectively. This paper considers applications where $c_{f}$ and $c_{g}$ are high, which limits the value of $N$ for a fixed onboard available computational ability. This yields a non-negligible Monte Carlo error that must be compensated.

\section{B. Application to constrained control under uncertainty}

An accurate knowledge of the failure probability $p_{f}$ is useful in many decision or control problems. For example, consider the following control problem. The objective is finding a future control sequence $\mathbf{u}_{k: K}$ that minimizes a cost function depending on the uncertain state trajectory $\mathbf{x}_{k: K}$ while ensuring that the output trajectory $\mathbf{z}_{k: K}$ satisfies some constraints:

$$
\mathbf{u}_{k: K}=\operatorname{argmin}\left(\varphi\left(\mathbf{x}_{k: K}, \mathbf{u}_{k: K}\right)\right) \text { s.t. } \mathbf{z}_{k: K}<\overline{\mathbf{Z}}
$$

where $\varphi: \mathbb{R}^{d(K-k+1)} \times \mathbb{R}^{d_{c}(K-k+1)} \rightarrow \mathbb{R}$ is a cost function and $\mathbf{x}_{k: K}$ Several approaches can be considered to solve the stochastic optimization (12). A way to approximate the solution is formulating it as an unconstrained deterministic scheme by including the failure probability $p_{f_{k}}=\mathbb{P}\left(\mathbf{z}_{k: K}>\right.$ $\overline{\mathbf{Z}})$ in the cost function to be minimized:

$$
\mathbf{u}_{k: K}=\operatorname{argmin}\left(J\left(\mathbf{x}_{k: K}, \mathbf{u}_{k: K}, p_{f_{k}}\right)\right)
$$


where $J: \mathbb{R}^{d(K-k+1)} \times \mathbb{R}^{d_{c}(K-k+1)} \times \mathbb{R} \rightarrow \mathbb{R}$ is the modified cost function. Note that chance constraint approaches [9] can also be used, but this is out of the scope of this paper.

The failure probability depends on the system dynamics $f$ and can be defined as a function of the control input $\mathbf{u}_{k: K}$ and of the current state $\mathbf{x}_{k}$. In what follows, we assume that $\mathbf{u}_{k: K}$ is determined by a single scalar parameter $\zeta_{k}$, i.e at each time-step, there exists a $\mathcal{C}^{2}$ function $\phi_{k}: \mathbb{R} \rightarrow \mathbb{R}^{d_{c}(K-k+1)}$ such that $\mathbf{u}_{k: K}=\phi_{k}\left(\zeta_{k}\right)$ :

$$
\begin{aligned}
\mathbb{R} \times \mathbb{R}^{d} & \rightarrow[0,1] \\
\left(\zeta_{k}, \mathbf{x}_{k}\right) & \mapsto p_{f_{k}}
\end{aligned}
$$

In practice, $\phi_{k}$ must be a priori defined. The control problem to solve becomes, for a given current state $\mathbf{x}_{k}$ :

$$
\zeta_{k}=\underset{\zeta}{\operatorname{argmin}}\left(J\left(\zeta, p_{f_{k}}\left(\zeta, \mathbf{x}_{k}\right)\right)\right)
$$

In this work, we will only consider costs of the form:

$$
\begin{aligned}
J:[\underline{\zeta}, \bar{\zeta}] \times \mathbb{R}^{d} & \rightarrow \mathbb{R} \\
\left(\zeta, \widehat{\mathbf{x}}_{k}\right) & \mapsto \kappa_{p}{\widehat{p_{f}}}_{k}\left(\zeta, \widehat{\mathbf{x}}_{k}\right)+\Delta \mathbf{x}^{T} \kappa_{x} \Delta \mathbf{x}
\end{aligned}
$$

where $\kappa_{p}>0$ and $\kappa_{x}>0, \Delta \mathbf{x}=\widehat{\mathbf{x}}_{k: K}-\mathbf{x}_{k: K}^{d}$ and $\mathbf{x}_{k: K}^{d}$ is the desired trajectory. In practice, the desired trajectory can be obtained using a deterministic offline optimization [16].

\section{ONLINE ESTIMATION OF THE FAILURE PROBABILITY}

A. Description of the two contributors to the failure probability error

In this section, we describe how the Monte Carlo failure probability estimation (11) behaves in terms of error with a state estimator in the loop. Two contributors to the error are identified:

- The state estimation filter, which provides the estimate state density $\widehat{p}\left(\mathbf{x}_{k} \mid \mathbf{Y}_{k}\right)$ and the output estimate density $\widehat{p}\left(\mathbf{z}_{k} \mid \mathbf{Y}_{k}\right)$. This density may be slightly different from the theoretical output density $p\left(\mathbf{z}_{k} \mid \mathbf{Y}_{k}\right)$. The error between the theoretical density and the estimate density has an impact on the prediction of the future output trajectory, and therefore on the failure probability estimation.

- The Monte Carlo formulation $p_{f}^{N}$ (11) of the failure probability implicates $N \in \mathbb{N}^{*}$ trajectory samples. This approximation has also an impact on the probability estimation.

Assume that the output Markov transition density verifies the following condition:

$$
\exists\left(\varepsilon_{k}, \lambda_{k}\right) \in \mathbb{R}^{+*} \times(0,1], \varepsilon_{k} \lambda_{k} \leq p\left(\mathbf{z}_{k} \mid \mathbf{z}_{k-1}\right) \leq \frac{1}{\varepsilon_{k}} \lambda_{k}
$$

Assume that the state process noise is bounded, i.e. $\forall k$ :

$$
\exists(\underline{\mathbf{w}}, \overline{\mathbf{w}}) \in \mathbb{R}^{d_{z}} \times \mathbb{R}^{d_{z}}, \forall \mathbf{w}_{k} \sim p\left(\mathbf{x}_{k} \mid \mathbf{x}_{k-1}\right), \underline{\mathbf{w}} \leq \mathbf{w}_{k} \leq \overline{\mathbf{w}}
$$

These assumptions are often verified in practice (e.g. with truncated Gaussian densities). Proposition 1 describes the characteristics of these two contributions to the total error.
Proposition 1: Consider the sample-based failure probability estimator (11). Then, the Monte Carlo failure probability estimation error $E=p_{f}-p_{f}^{N}$ is impacted by two contributors:

$$
E=\epsilon+\epsilon^{N}
$$

where:

- $\epsilon$ is the impact of the current state estimation filter error $\widehat{\mathbf{x}}_{k}-\mathbf{x}_{k}$. If assumptions (17) and (18) are satisfied, it is bounded by:

$$
|\epsilon| \leq \frac{2 \tau^{K-k}}{\varepsilon^{2} \log (3)} d_{k}(p, \widehat{p})
$$

where the metrics $d_{k}(p, \widehat{p})$ quantifies the density estimation error at time-step $k$ and is defined by (27), $\varepsilon_{k} \geq \varepsilon>0$, and $\tau=\left(1-\varepsilon^{2}\right) /\left(1+\varepsilon^{2}\right)$;

- $\epsilon^{N}$ is the impact of the Monte Carlo approximation (11), a.s. bounded by:

$$
\left|\epsilon^{N}\right| \leq 3 \sqrt{\frac{\tilde{p}_{f}\left(1-\tilde{p}_{f}\right)}{N}}
$$

Proof: Define $E=p_{f}-p_{f}^{N}$ the error made on the Monte Carlo failure probability estimate $p_{f}^{N}$ with respect to the actual probability $p_{f}$ obtained with an optimal navigation filter and an exhaustive failure prediction process. $E$ can be rewritten by introducing a virtual failure probability estimator $\tilde{p}_{f}$, obtained using the theoretical output prediction from the current state density estimate $\widehat{p}\left(\mathbf{x}_{k} \mid \mathbf{Y}_{k}\right)$ :

$$
E=p_{f}-\tilde{p}_{f}+\tilde{p}_{f}-p_{f}^{N}=\epsilon+\epsilon^{N}
$$

where $\epsilon=p_{f}-\tilde{p}_{f}$ represents the contribution of the state estimation filter to the error and $\epsilon^{N}=\tilde{p}_{f}-p_{f}^{N}$ is the error of the Monte Carlo approximation.

To describe $\epsilon$, it is possible to project the failure probability calculation to the terminal predicted time-step $K: p_{f}=\mu_{p\left(\mathbf{z}_{K} \mid \mathbf{Y}_{k}\right)}(\mathcal{D})$ and $\tilde{p}_{f}=\mu_{\widehat{p}\left(\mathbf{z}_{K} \mid \mathbf{Y}_{k}\right)}(\mathcal{D})$ where $\mathcal{D} \subset \mathbb{R}^{d_{z}}$ is the projection of the trajectory constraint set $\left\{\mathbf{z}_{k: K} \in \mathbb{R}^{d_{z}(K-k+1)} \mid \mathbf{z}>\overline{\mathbf{Z}}\right\}$ to the last output set:

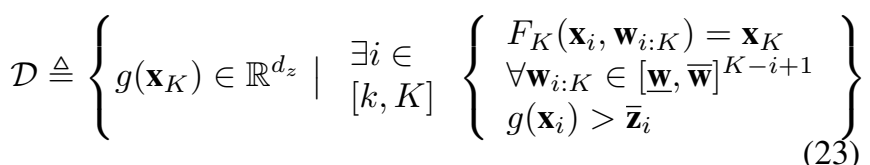

In other words, $\mathcal{D}$ contains all the terminal outputs yielded by non-admissible trajectories. If assumption (17) is satisfied, the following inequality holds ([17], Theorem 2.21):

$$
\begin{aligned}
& \sup _{\mathcal{A}}\left|\mu_{p\left(\mathbf{z}_{K} \mid \mathbf{Y}_{k}\right)}(\mathcal{A})-\mu_{\widehat{p}\left(\mathbf{z}_{K} \mid \mathbf{Y}_{k}\right)}(\mathcal{A})\right| \leq \\
& \frac{2 \tau^{K-k}}{\varepsilon^{2} \log (3)} \sup _{\mathcal{A}}\left|\mu_{p\left(\mathbf{z}_{k} \mid \mathbf{Y}_{k}\right)}(\mathcal{A})-\mu_{\widehat{p}\left(\mathbf{z}_{k} \mid \mathbf{Y}_{k}\right)}(\mathcal{A})\right|
\end{aligned}
$$

where the sup is taken over all the open subsets $\mathcal{A} \subset \mathbb{R}^{d_{z}}$, $\varepsilon_{k} \geq \varepsilon>0$, and $\tau=\left(1-\varepsilon^{2}\right) /\left(1+\varepsilon^{2}\right)$. By definition, domain $\mathcal{D}$ is a just a special case of one open subset of $\mathbb{R}^{d_{z}}$, which yields:

$$
\left|p_{f}-\tilde{p}_{f}\right| \leq \sup _{\mathcal{A}}\left|\mu_{p\left(\mathbf{z}_{K} \mid \mathbf{Y}_{k}\right)}(\mathcal{A})-\mu_{\widehat{p}\left(\mathbf{z}_{K} \mid \mathbf{Y}_{k}\right)}(\mathcal{A})\right|
$$


and then

$$
|\epsilon| \leq \frac{2 \tau^{K-k}}{\varepsilon^{2} \log (3)} d_{k}(p, \widehat{p})
$$

where the metrics $d_{k}(p, \widehat{p})$ quantifies the density estimation error of output $\mathbf{z}_{k}$ at time-step $k$ :

$$
d_{k}(p, \widehat{p}) \triangleq \sup _{\mathcal{A}}\left|\mu_{p\left(\mathbf{z}_{k} \mid \mathbf{Y}_{k}\right)}(\mathcal{A})-\mu_{\widehat{p}\left(\mathbf{z}_{k} \mid \mathbf{Y}_{k}\right)}(\mathcal{A})\right|
$$

which yields (20). The density error $d_{k}(p, \widehat{p})$ is directly linked with the state estimation error, and therefore quantifies the state estimator contribution to the failure probability error.

Since $p_{f}^{N}=\frac{1}{N} \sum_{i} \mathbf{1}_{\{\mathbf{z}>\overline{\mathbf{z}}\}}$, it can be interpreted as the sum of $N$ Bernoulli variables (that have a probability $\tilde{p}_{f}$ to be equal to $1 / N)$, which yields:

$$
\operatorname{Var}\left(\epsilon^{N}\right)=\frac{\tilde{p}_{f}\left(1-\tilde{p}_{f}\right)}{N}+\left(\tilde{p}_{f}-\tilde{p}_{f}\right)^{2}=\frac{\tilde{p}_{f}\left(1-\tilde{p}_{f}\right)}{N}
$$

Then, for $N$ sufficiently high, the Monte Carlo error $\epsilon^{N}$ can be a.s. bounded by:

$$
\left|\epsilon^{N}\right| \leq 3 \sqrt{\operatorname{Var}\left(\epsilon^{N}\right)}
$$

which yields (21).

B. Gaussian filtering of the noised failure probability measure

As shown in Section III-A, the failure probability $p_{f}$ cannot be accurately estimated because of two sources of error: the state estimation error, and the Monte Carlo discrete approximation. The objective of this section is refining the estimation of $p_{f}$ despite the errors introduced by the navigation filter and Monte Carlo algorithm. Define the actual failure probability $p_{f}$ as a Markov process, i.e. for each timestep $k$ :

$$
p_{f_{k}}=p_{f_{k-1}}+\omega_{k}
$$

where $\omega_{k}$ is a noise of variance $\Omega \in \mathbb{R}^{+*}$. Define $p_{f_{k}}^{N}$ as a pseudo-measurement of $p_{f_{k}}$ :

$$
p_{f k}^{N}=p_{f_{k}}+v_{k}
$$

where $v_{k}$ is a noise of variance $V_{k}$ and is linked to the error $E$ described by Proposition 1. In practice $V_{k}$ can be obtained by an empirical variance estimator. An unbiased estimate of $p_{f_{k}}$ can be obtained by performing a recursive least square estimation:

$$
\begin{gathered}
{\widehat{p_{f}}}_{k}=\Sigma_{k}\left(\frac{1}{\Sigma_{k \mid k-1}}{\widehat{p_{f}}}_{k-1}+\frac{1}{V_{k}} p_{f_{k}}^{N}\right) \\
\Sigma_{k}=\left(\frac{1}{\Sigma_{k \mid k-1}}+\frac{1}{V_{k}}\right)^{-1} \\
\Sigma_{k \mid k-1}=\Sigma_{k-1}+\Omega
\end{gathered}
$$

The computational cost of process (32) is of $\mathcal{O}(10)$ elementary operations, which is usually neglectible with respect to the cost of $p_{f}^{N}$ (see Section II-A.3).

Proposition 2: Assume that ${\widehat{p_{f}}}_{k}$ is recursively obtained using (32). Then, its associated density function converges in distribution to a Gaussian function and:

$$
\lim _{k \rightarrow \infty}\left({\widehat{p_{f}}}_{k}-p_{f_{k}}\right)=0
$$

Proof: The least square recursive process (32) is equivalent to a linear scalar Kalman Filter [18]. According to Aliev [19], the estimation error converges if there exists $0<\beta<\infty$ and $0<\delta \leq 1$ such that:

$$
\left\{\begin{array} { c } 
{ \Sigma _ { 0 } \leq \beta } \\
{ \Omega \leq \beta } \\
{ V _ { k } \leq \beta }
\end{array} \text { and } \left\{\begin{array}{c}
\mathbb{E}\left(\left|\omega_{k}\right|^{2+\delta}\right)<\beta \\
\mathbb{E}\left(\left|v_{k}\right|^{2+\delta}\right)<\beta \\
\mathbb{E}\left(\left|{\widehat{p_{f}}}_{f_{0}}-p_{f_{0}}\right|^{2+\delta}\right)<\beta
\end{array}\right.\right.
$$

In our case, the random variable to estimate is bounded by $0 \leq p_{f} \leq 1$. Therefore, the estimate value is constrained by $0 \leq{\widehat{p_{f}}}_{k} \leq 1$ (it can be initialized to $0<\widehat{p_{f}}<1$ ) and the model noises are necessarily lower than unity, i.e., $\left|\omega_{k}\right|<1$, $\left|v_{k}\right|<1$, and $\left|\widehat{p_{f}}-p_{f_{0}}\right|<1$. Therefore, the initial variance and the process noise variance can be bounded to $\Sigma_{0} \leq 1$ and $V_{k} \leq 1$. Since $0 \leq p_{f_{k}} \leq 1$, the Monte Carlo measure of $p_{f_{k}}$ is also bounded to $0 \leq p_{f_{k}}^{N} \leq 1$, which yields $V_{k} \leq 1$. Then, one obtains the left system in (34) with $\beta=1$. Since $\left|\omega_{k}\right|<1,\left|v_{k}\right|<1$ and $\left|\widehat{p_{f_{0}}}-p_{f_{0}}\right|<1$, one obtains with any $\delta \geq 0$ :

$$
\left\{\begin{array}{c}
\left|\omega_{k}\right|^{2+\delta}<1 \\
\left|v_{k}\right|^{2+\delta}<1 \\
\left|\widehat{p}_{f_{0}}-p_{f_{0}}\right|^{2+\delta}<1
\end{array}\right.
$$

By taking the expectancy of both hand sides, one obtains (34) with $\beta=1$. Therefore, the models (30) and (31) satisfy assumptions (34), which allow the estimator (32) to converge to $p_{f_{k}}$.

\section{Application to CONSTRAined CONTROL UNDER UNCERTAINTY}

Section III-B introduced a computationally efficient method to estimate the probability of failure $p_{f_{k}}$ along the future trajectory (from time-step $k$ to a given horizon $K$ ). In order to illustrate an application of this approach, this section describes two ways to determine a sequence of future control inputs $\mathbf{u}_{k: K}$ that minimizes the failure probability $p_{f}$.

As introduced in Sections II and III, the failure probability $p_{f_{k}}$ and the state $\mathbf{x}_{k}$ are stochastic processes that must be estimated in an iterative way. Problem (15) becomes:

$$
\widehat{\zeta_{k}}=\underset{\zeta}{\operatorname{argmin}}\left(J\left(\zeta,{\widehat{p_{f}}}_{k}\left(\zeta, \widehat{\mathbf{x}}_{k}\right)\right)\right)
$$

\section{A. Failure probability minimization by local gradient descent}

A way to approach the solution of Problem (36) is solving the following equation:

$$
\left.\Psi\left(\zeta, \widehat{\mathbf{x}}_{k}\right) \triangleq \frac{\partial J(\zeta, \mathbf{x})}{\partial \zeta}\right|_{\mathbf{x}=\widehat{\mathbf{x}}_{k}}=0
$$

assuming that $J$ is locally convex. An online accurate solving of Problem (37) would be highly computationally demanding. Several approaches were proposed in the literature (e.g. online minimization [20] [21] [22]), but only for linear dynamics and constraints. It is nevertheless possible to approach the solution in a time-iterative way by a local gradient descent:

$$
\tilde{\zeta}_{k}=-\kappa_{p} \Psi\left(\zeta_{k-1}, \widehat{\mathbf{x}}_{k}\right)-\kappa_{x}\left(\widehat{\mathbf{x}}_{k: K}-\mathbf{x}_{k: K}^{d}\right)
$$

where $\kappa_{p}>0$ and $\kappa_{x}>0$. 
The gradient of the failure probability can be estimated by finite difference using two estimators ${\widehat{p_{f}}}_{k}\left(\tilde{\zeta}_{k-1}-\delta \zeta, \widehat{\mathbf{x}}_{k}\right)$ and ${\widehat{p_{f}}}_{k}\left(\tilde{\zeta}_{k-1}+\delta \zeta, \widehat{\mathbf{x}}_{k}\right)$ by:

$\Psi\left(\tilde{\zeta}_{k-1}, \widehat{\mathbf{x}}_{k}\right) \approx \frac{1}{2 \delta \zeta}\left({\widehat{p_{f}}}_{k}\left(\tilde{\zeta}_{k-1}+\delta \zeta, \widehat{\mathbf{x}}_{k}\right)-{\widehat{p_{f}}}_{k}\left(\tilde{\zeta}_{k-1}-\delta \zeta, \widehat{\mathbf{x}}_{k}\right)\right.$

where $\delta \zeta>0$ and ${\widehat{p_{f}}}_{k}\left(\tilde{\zeta}_{k-1}-\delta \zeta, \widehat{\mathbf{x}}_{k}\right)$ and ${\widehat{p_{f}}}_{k}\left(\tilde{\zeta}_{k-1}+\delta \zeta, \widehat{\mathbf{x}}_{k}\right)$ are both obtained with the formulation (32). They represent the least square estimations of the two following Markov processes:

$$
\begin{aligned}
& p_{f_{k}}\left(\tilde{\zeta}_{k-1}+\delta \zeta, \mathbf{x}_{k}\right)=p_{f_{k-1}}\left(\tilde{\zeta}_{k-1}+\delta \zeta, \mathbf{x}_{k-1}\right)+\omega_{k}^{1} \\
& p_{f_{k}}\left(\tilde{\zeta}_{k-1}-\delta \zeta, \mathbf{x}_{k}\right)=p_{f_{k-1}}\left(\tilde{\zeta}_{k-1}-\delta \zeta, \mathbf{x}_{k-1}\right)+\omega_{k}^{2}
\end{aligned}
$$

associated to the two following Monte Carlo measures:

$$
\begin{aligned}
& p_{f_{k}}^{N}\left(\tilde{\zeta}_{k-1}+\delta \zeta, \widehat{\mathbf{x}}_{k}\right)=p_{f_{k}}\left(\tilde{\zeta}_{k-1}+\delta \zeta, \mathbf{x}_{k}\right)+v_{k}^{1} \\
& p_{f_{k}}^{N}\left(\tilde{\zeta}_{k-1}-\delta \zeta, \widehat{\mathbf{x}}_{k}\right)=p_{f_{k}}\left(\tilde{\zeta}_{k-1}-\delta \zeta, \mathbf{x}_{k}\right)+v_{k}^{2}
\end{aligned}
$$

where $\omega_{k}^{1}$ and $\omega_{k}^{2}$ are noises of variance $\Omega$, and $v_{k}^{1}$ and $v_{k}^{2}$ are noises of variance $V_{k}$ that account for state estimation and Monte Carlo uncertainties. Note that the approached solution $\tilde{\zeta}_{k}$ of Problem (36) remains sub-optimal (the optimality is out of the scope of this paper). The computation load of the gradient descent control is about $\mathcal{O}\left(N(K-k)\left(c_{f}+c_{g}\right)\right)$ with $c_{f}>0$ and $c_{g}>0$ the cost of the dynamical transition $f$ and the output computation $g$ respectively.

\section{B. Failure probability minimization by grid search}

Another way to approach the solution to Problem (36) is evaluating a collection of $M \in \mathbb{N}^{*}$ stochastic processes $p_{f_{k}}\left(\zeta^{i}, \widehat{\mathbf{x}}_{k}\right)$, on a grid of control sequences: $\underline{\zeta} \leq \zeta^{1}<\ldots<$ $\zeta^{M} \leq \bar{\zeta}, \forall i=\{1, \ldots, M\}$ :

$$
\begin{gathered}
p_{f_{k}}\left(\zeta^{1}, \mathbf{x}_{k}\right)=p_{f_{k-1}}\left(\zeta^{1}, \mathbf{x}_{k-1}\right)+\omega_{k} \\
\vdots \\
p_{f_{k}}\left(\zeta^{M}, \mathbf{x}_{k}\right)=p_{f_{k-1}}\left(\zeta^{M}, \mathbf{x}_{k-1}\right)+\omega_{k}
\end{gathered}
$$

associated to the $M$ following Monte Carlo measures:

$$
\begin{gathered}
p_{f_{k}}^{N}\left(\zeta^{1}, \widehat{\mathbf{x}}_{k}\right)=p_{f_{k}}\left(\zeta^{1}, \mathbf{x}_{k}\right)+v_{k} \\
\vdots \\
p_{f_{k}}^{N}\left(\zeta^{M}, \widehat{\mathbf{x}}_{k}\right)=p_{f_{k}}\left(\zeta^{M}, \mathbf{x}_{k}\right)+v_{k}
\end{gathered}
$$

where $\omega_{k}$ and $v_{k}$ are noises of variances $\Omega$ and $V_{k}$. Then, a cost function $J(\zeta, \mathbf{x})$ can be approximated on the discrete control domain $\left\{\zeta^{1}, \ldots, \zeta^{M}\right\} \subset[\zeta, \bar{\zeta}]$. The computation of the grid search control is about $\mathcal{O}\left(M N(K-k)\left(c_{f}+c_{g}\right)\right)$.

\section{NUMERICAL RESULTS}

\section{A. Space X's Starship-like vehicle reentry model}

In September 2018, a novel design for a reusable atmospheric reentry vehicle was proposed: Space X's Starship (SXS [15]). Its main difference with previous similar systems lays in its aerodynamic actuators that allow for stable atmospheric flight at very high angles of attack (AoA), up to $90^{\circ}$ forward and rearward. The large range of reachable AoA values brings new perspectives to reentry control. However, in the presence of state uncertainty, the risk of mechanical constraints violation may not be easily predictable offline, which motivates the use of online failure probability estimator. The AoA can be determined by two sets of flat surfaces (front and aft fins) able to rotate along the roll axis (see Figure 1, realized using OpenVSP [23]). We use here a 3DoF simulation model of a SXS-like vehicle [24] controlled by its AoA $\alpha \in \mathbb{R}$. The vehicle's evolution is defined in its discrete form by:

$\mathbf{x}_{k}=\mathbf{x}_{k-1}+\left[\begin{array}{c}\Delta t V_{k-1} \cos \gamma_{k-1} \\ \Delta t V_{k-1} \sin \gamma_{k-1} \\ -\frac{\Delta t}{2 m} \rho S V_{k-1}^{2} C_{x}\left(V_{k-1}, \alpha\right)-\Delta t g \sin \gamma_{k-1} \\ \frac{\Delta t}{2 m} \rho S V_{k-1} C_{z}\left(V_{k-1}, \alpha\right)-\frac{\Delta t}{V_{k-1}} g \cos \gamma_{k-1}\end{array}\right]+\mathbf{w}_{k}$

where $\mathbf{x}_{k}=\left[x_{k}, z_{k}, V_{k}, \gamma_{k}\right]^{T}$ is the state which consists of the linear position, the ground altitude, the vehicle speed and the path angle, $\Delta t=1 \mathrm{~s}$ the integration time-step, $g$ the local gravity field, $m=85000 \mathrm{~kg}$ the vehicle's mass, $\mathbf{w}_{k}$ a Gaussian process noise, and $\left(C_{x}, C_{z}\right)$ the aerodynamic coefficients [24], depending on the Mach number and the AoA $\alpha$. The local atmosphere density is defined by $\rho=$ $\rho_{0} e^{-z / h_{s}}$ with $\rho_{0}=1.225 \mathrm{~kg} / \mathrm{m}^{-3}$ and $h_{s}=7143$ $\mathrm{m}$. During the reentry phase, an Inertial Measurement Unit (IMU) provides accelerations and angular rate data. We assume that measurements of $V_{k}$ and $\gamma$ can be obtained by integration, which yields the following linear measurement equation:

$$
\mathbf{y}_{k}=\left[\begin{array}{llll}
0 & 0 & 1 & 0 \\
0 & 0 & 0 & 1
\end{array}\right] \mathbf{x}_{k}+\mathbf{v}_{k}
$$

where the measurement noise $\mathbf{v}_{k} \in \mathbb{R}^{2}$ is assumed to be Gaussian. The state estimation is performed by an Extended Kalman Filter. The constraints are on the thermal flow $\Phi$, the dynamic pressure $P$, and the load factor $\Gamma$ :

$$
\mathbf{z}_{k}=[\Phi, P, \Gamma]^{T} \leq \overline{\mathbf{z}}
$$

where

$$
\Phi=C_{q} \sqrt{\rho} V^{3} \quad P=\frac{1}{2} \rho V^{2} \quad \Gamma=\frac{S}{2 m} \rho V^{2} C_{x} \sqrt{1+\left(\frac{C_{z}}{C_{x}}\right)^{2}}
$$

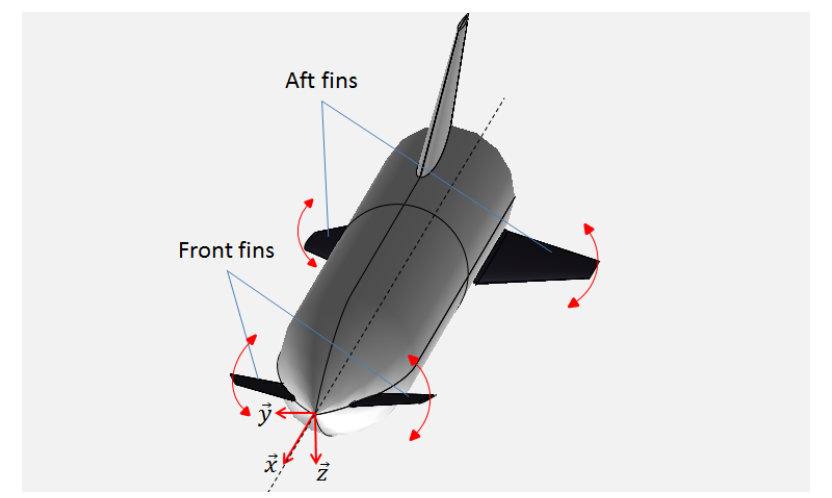

Fig. 1. SXS 3D OpenVSP model 
TABLE I

ESTIMATION PERFORMANCE FOR $p_{f}^{N}, \tilde{p}_{f}$ AND $\widehat{p}_{f}$

\begin{tabular}{c|c|c|c}
$N$ & 100 & 1000 & 5000 \\
\hline Mean RMSE for $p_{f}^{N}$ & 0.0717 & 0.0678 & 0.0671 \\
Mean RMSE for $\tilde{p}_{f}$ & 0.0698 & 0.0698 & 0.0698 \\
Mean RMSE for $\widehat{p}_{f}$ & 0.0482 & 0.0442 & 0.0442
\end{tabular}

where $C_{q}=1.710^{-4}$ S.I. The constraint bound is defined by $\overline{\mathbf{z}}=[\bar{\Phi}, \bar{P}, \bar{\Gamma}]^{T}$ with $\bar{\Phi}=375100 \mathrm{~W} / \mathrm{m}^{2}, \bar{P}=5000 \mathrm{kPa}$, $\bar{\Gamma}=3.5 \mathrm{~g}$. The AoA is constrained between $45^{\circ}$ and $90^{\circ}$ and the future AoA control sequence is assumed to be constant during the trajectory prediction: $\alpha_{k: K}=\alpha_{k}$. The state is initialized to $\mathbf{x}_{0}=\left[0 \mathrm{~m}, 90 \mathrm{~km}, 7400 \mathrm{~m} / \mathrm{s}, 0^{\circ}\right]^{T}$ with a Gaussian uncertainty of standard deviation (std) $[10,10,5,0.05]^{T}$. The process noise in dynamics (1) has a std of $[1000,50,20,0.1]^{T}$ and the measurement noise has a std of $\left[1 \mathrm{~m} / \mathrm{s}, 0.5^{\circ}\right]$.

\section{B. Numerical results}

The following results are obtained on $n=50$ runs. For each run, the initial state estimate, process noise and measurement noise samples are drawn randomly, which covers a variety of simulation cases. Define the Root Mean Square Error criterion on $n$ runs by $\operatorname{RMSE}_{k}\left(a_{k}, b_{k}\right)=\sqrt{\sum_{i=1}^{n}\left(a_{k}-b_{k}\right)^{2} / n}$ where $a_{k}, b_{k} \in$ $\mathbb{R}$.

1) Failure probability estimation: We first evaluate the failure probability estimation error. Table I shows the average RMSE of the naive Monte Carlo estimation $\operatorname{RMSE}_{k}\left(p_{f}^{N}, p_{f}\right)$, the Kalman biased estimation $\operatorname{RMSE}_{k}\left(\tilde{p}_{f}, p_{f}\right)$ and the filtered estimation $\operatorname{RMSE}_{k}\left(\widehat{p}_{f}, p_{f}\right)$, where $p_{f}$ and $\tilde{p}_{f}$ are evaluated on a benchmark set of $N_{b}=$ $10^{4}$ trajectories. The random walk (30) is assumed to have a standard deviation (std) of 0.01 and the Monte Carlo measure (31) std is computed empirically using the 50 last Monte Carlo measures. The trajectory lasts for $200 \mathrm{~s}$ and $p_{f}=0.01$. The accuracy of $p_{f}^{N}$ and $\widehat{p}_{f}$ increases with $N$ (note that $\tilde{p}_{f}$ does not depend on $N$ since it is evaluated on $N_{b}$ trajectories). The filtered $\widehat{p}_{f}$ (32) makes it possible to reduce the RMSE by $33 \%$ with respect to the naive Monte Carlo probability $p_{f}^{N}$. However, note that although Proposition 2 proves that $\widehat{p}_{f}$ tends to $p_{f}$, the estimate remains biased (overestimation). This can be explained by the fact that the noise $v_{k}$ in (31) is not centered. Therefore, we can expect some conservatism in the following control application.

2) Application to constrained reentry under state uncertainty: In this section, we illustrate the behavior of the gradient descent approach (Section IV-A) and the grid search approach (Section IV-B) on the control problem introduced earlier. The trajectory lasts until the spacecraft falls below the altitude of $10 \mathrm{~km}$. The desired longitudinal coordinate at this point is $x_{d}=3500 \mathrm{~km}$ from the starting point. At each timestep, the number of sampled trajectories is fixed to $N=100$, with a receding prediction horizon of $K=k+100 \mathrm{~s}$. Table II shows the averaged results on $n=50$ runs. The two methods are compared to a deterministic Model Predictive Control (classic MPC [25]) approach that solves Problem (12) using
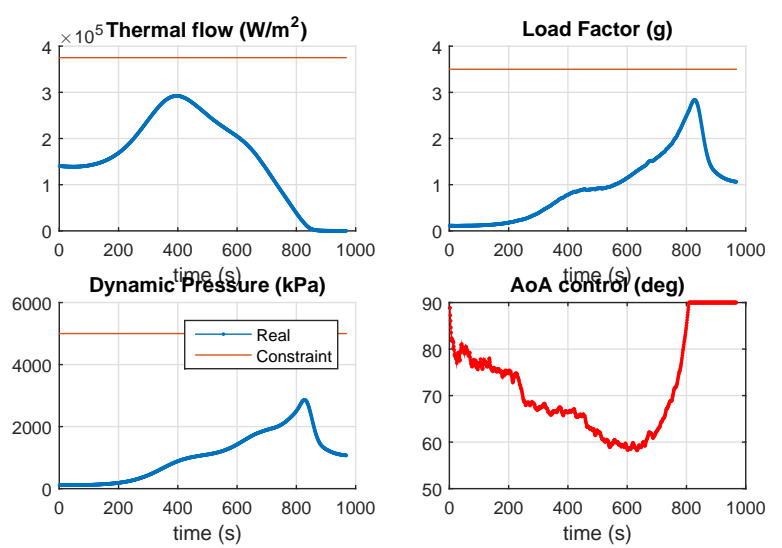

Fig. 2. Simulation example for Earth reentry: constraints satisfaction and control input by gradient descent (see Section IV-A)

TABLE II

AOA CONTROL PERFORMANCE

\begin{tabular}{c|c|c|c} 
& Gradient descent & Grid search & MPC \\
\hline Actual $p_{f}$ & 0 & 0 & 0.12 \\
Tracking RMSE $(\mathrm{km})_{\text {Computation time }^{1}(\mathrm{~s})}$ & 171 & 182 & 1.2 \\
1. Time to compute one control sequence, on a 1.6GHz CPU running Matlab & 8.9 & 7.8
\end{tabular}

the expectancy of the predicted trajectory and outputs. The actual $p_{f}$ represents here the actual proportion of runs leading to a spacecraft crash (i.e constraint violation). The proposed methods, by including the minimization of $p_{f}$ in the control cost (36) never yield non-admissible trajectories $\left(p_{f}=0\right)$. The MPC, that only accounts for the mean value of uncertain parameters, yields a $12 \%$ of non-admissible trajectory (i.e. potentially fatal failures for the system). As expected, the tracking error $\operatorname{RMSE}_{k}\left(x_{k f}, x_{d}\right)$, that quantifies the error between the reached final position $(k=k f)$ and the desired position shows some conservatism for the proposed methods, while deterministic MPC is more accurate. Figure 2 illustrates the AoA control input and constrained output profiles for the gradient descent case. Before $t=700 \mathrm{~s}$, the vehicle progressively decreases its AoA to lower the failure probability while producing enough drag force to attempt to reach its desired final position. In particular, between $300 \mathrm{~s}$ and $700 \mathrm{~s}$, the vehicle enters the dense atmosphere with an important velocity, which involves a high risk of constraints violation. After $t=700 \mathrm{~s}$, the vehicle can fly with a higher AoA.

\section{CONCLUSION}

Determining online the probability that a system does not satisfy mechanical or physical constraints is crucial in the aerospace field. This paper studies the impact of both state estimation error and computational load limitation on the quality of the failure probability estimation via Monte Carlo sampling. The impact of these two aspects is first theoretically described in terms of error bounds. A refining estimator is then introduced, based on least square estimation. The least square failure probability is theoretically shown to be convergent and demonstrated to significantly reduce the estimation error in simulation. The use of this 
method is illustrated on a state constrained control application for Earth reentry. It is demonstrated to bring robustness to uncertainty, although yielding some conservatism. Future work will include a corrective strategy to compensate the bias of the Monte Carlo failure probability measure, which should further reduce the filtered estimation error. Furthermore, a more realistic formulation of the control application will be considered, including the online tracking of an a priori defined optimal trajectory.

\section{REFERENCES}

[1] A. Harbitz, "An efficient sampling method for probability of failure calculation," Structural safety, vol. 3, no. 2, pp. 109-115, 1986.

[2] J. Morio, "Influence of input pdf parameters of a model on a failure probability estimation," Simulation Modelling Practice and Theory, vol. 19, no. 10, pp. 2244-2255, 2011.

[3] S. Lacaze, L. Brevault, S. Missoum, and M. Balesdent, "Probability of failure sensitivity with respect to decision variables," Structural and Multidisciplinary Optimization, vol. 52, no. 2, pp. 375-381, 2015.

[4] V. Chabridon, M. Balesdent, J.-M. Bourinet, J. Morio, and N. Gayton, "Evaluation of failure probability under parameter epistemic uncertainty: application to aerospace system reliability assessment," Aerospace Science and Technology, vol. 69, pp. 526-537, 2017.

[5] L. P. Kaelbling, M. L. Littman, and A. R. Cassandra, "Planning and acting in partially observable stochastic domains," Artificial intelligence, vol. 101, no. 1, pp. 99-134, 1998.

[6] A. T. Schwarm and M. Nikolaou, "Chance-constrained model predictive control," AIChE Journal, vol. 45, no. 8, pp. 1743-1752, 1999.

[7] L. Blackmore, H. Li, and B. Williams, "A probabilistic approach to optimal robust path planning with obstacles," in American Control Conference, 2006. IEEE, 2006, pp. 7-pp.

[8] G. C. Calafiore and L. El Ghaoui, "On distributionally robust chanceconstrained linear programs," Journal of Optimization Theory and Applications, vol. 130, no. 1, pp. 1-22, 2006.

[9] L. Blackmore, M. Ono, A. Bektassov, and B. C. Williams, "A probabilistic particle-control approximation of chance-constrained stochastic predictive control," IEEE transactions on Robotics, vol. 26, no. 3, pp. 502-517, 2010.

[10] A. Mesbah, S. Streif, R. Findeisen, and R. D. Braatz, "Stochastic nonlinear model predictive control with probabilistic constraints," in American Control Conference (ACC), 2014 . IEEE, 2014, pp. 24132419.

[11] R. Huang, S. C. Patwardhan, and L. T. Biegler, "Robust stability of nonlinear model predictive control with extended kalman filter and target setting," International Journal of Robust and Nonlinear Control, vol. 23, no. 11, pp. 1240-1264, 2013.

[12] S. P. Meyn and R. L. Tweedie, Markov chains and stochastic stability. Springer Science \& Business Media, 2012.

[13] C. Robert and G. Casella, Monte Carlo statistical methods. Springer Science \& Business Media, 2013.

[14] J. Morio and M. Balesdent, Estimation of rare event probabilities in complex aerospace and other systems: a practical approach. Woodhead Publishing, 2015.

[15] SpaceX. (2018) Spacex website. [Online]. Available: https://www.spacex.com

[16] E. Trélat, "Optimal control of a space shuttle and numerical simulations," Discrete and Continuous Dynamical Systems-Series S, pp. 842-851, 2003.

[17] N. Oudjane, "Stabilité et approximations particulaires en filtrage non linéaire application au pistage," Ph.D. dissertation, Rennes 1, 2000.

[18] A. Jazwinski, "Stochastic and filtering theory. mathematics in sciences and engineering series, series 64," 1970.

[19] F. A. Aliev and L. Ozbek, "Evaluation of convergence rate in the central limit theorem for the kalman filter," IEEE Transactions on Automatic Control, vol. 44, no. 10, pp. 1905-1909, 1999.

[20] Y. Wang and S. Boyd, "Fast model predictive control using online optimization," IEEE Transactions on control systems technology, vol. 18, no. 2, pp. 267-278, 2010.

[21] J. L. Jerez, P. J. Goulart, S. Richter, G. A. Constantinides, E. C. Kerrigan, and M. Morari, "Embedded online optimization for model predictive control at megahertz rates," IEEE Transactions on Automatic Control, vol. 59, no. 12, pp. 3238-3251, 2014.
[22] P. Patrinos and A. Bemporad, "An accelerated dual gradient-projection algorithm for embedded linear model predictive control," IEEE Transactions on Automatic Control, vol. 59, no. 1, pp. 18-33, 2014.

[23] W. Fredericks, K. Antcliff, G. Costa, N. Deshpande, M. Moore, E. San Miguel, and A. Snyder, "Aircraft conceptual design using vehicle sketch pad," in 48th AIAA Aerospace Sciences Meeting Including the New Horizons Forum and Aerospace Exposition, 2010, p. 658.

[24] T. Cantou, N. Merlinge, and R. Wuilbercq, "3dof simulation model and specific aerodynamic control capabilities for a space x's starshiplike atmospheric reentry vehicle," in EUCASS 2019 (submitted), 2019, pp. EUCASS-Paper.

[25] M. Morari and J. H. Lee, "Model predictive control: past, present and future," Computers and Chemical Engineering, vol. 23, no. 4-5, pp. $667-682,1999$. 